\title{
Direct evidence for cycloidal modulations in the thermal-fluctuation-stabilized spin spiral and skyrmion states of $\mathrm{GaV}_{4} \mathrm{~S}_{8}$
}

\author{
J. S. White,,${ }^{1, *}$ Á. Butykai, ${ }^{2}$ R. Cubitt,${ }^{3}$ D. Honecker, ${ }^{3}$ C. D. Dewhurst,${ }^{3}$ L. F. Kiss, ${ }^{4}$ V. Tsurkan,${ }^{5,6}$ and S. Bordács ${ }^{2, \dagger}$ \\ ${ }^{1}$ Laboratory for Neutron Scattering and Imaging (LNS), Paul Scherrer Institut (PSI), CH-5232 Villigen, Switzerland \\ ${ }^{2}$ Department of Physics, Budapest University of Technology and Economics and MTA-BME Lendület Magneto-optical \\ Spectroscopy Research Group, H-1111 Budapest, Hungary \\ ${ }^{3}$ Institut Laue-Langevin, 71 avenue des Martyrs, CS 20156, F-38042 Grenoble Cedex 9, France \\ ${ }^{4}$ Department of Experimental Solid State Physics, Institute for Solid State Physics and Optics, \\ Wigner-MTA Research Centre for Physics, H-1121 Budapest, Hungary \\ ${ }^{5}$ Experimental Physics V, Center for Electronic Correlations and Magnetism, University of Augsburg, D-86135 Augsburg, Germany \\ ${ }^{6}$ Institute of Applied Physics, Academy of Sciences of Moldova, Academiei strada 5, Chisinau, Republic of Moldova
}

(Received 18 April 2017; revised manuscript received 20 September 2017; published 5 January 2018)

\begin{abstract}
We report small-angle neutron scattering studies of the lacunar spinel $\mathrm{GaV}_{4} \mathrm{~S}_{8}$, which reveal the long-wavelength magnetic phases to be cycloidally modulated. Upon cooling, these modulated phases, including a recently proposed Néel-type skyrmion phase, transform into a simple ferromagnetic state. These results indicate the modulated phases in $\mathrm{GaV}_{4} \mathrm{~S}_{8}$ gain their stability from thermal fluctuations, while at lower temperatures the ferromagnetic state emerges in accord with the strong easy-axis magnetic anisotropy. Crucially, our study provides microscopic evidence that the skyrmions in $\mathrm{GaV}_{4} \mathrm{~S}_{8}$ indeed display a Néel-type helicity. More generally, our approach can be applied to evidence the helicity of any bulk skyrmion phase.
\end{abstract}

DOI: 10.1103/PhysRevB.97.020401

Particlelike magnetic skyrmions with topologically nontrivial spin textures receive continued attention since their creation, stability, and annihilation, either individually or in skyrmion lattices (SkLs), raise fundamental questions related to topology in physics [1-3]. Moreover, the observations of metastable skyrmions [4,5] and their current-driven dynamics [6,7] inspire new ideas for using skyrmions in data storage and logic devices [8-10].

The skyrmion spin texture can be described by a vector field $\mathbf{S}=\mathrm{S}\{\cos [\Phi(\varphi)] \sin [\Theta(\rho)], \sin [\Phi(\varphi)] \sin [\Theta(\rho)]$, $\cos [\Theta(\rho)]\}$, where $\varphi$ and $\rho$ are the azimuth and radial spatial coordinates, respectively $[8,11]$. The nontrivial skyrmion topology is evidenced when its spin pattern is mapped onto a sphere, and the mapping wraps the surface of the sphere entirely. Two parameters classify the skyrmion type: the vorticity $m$, which is a nonzero integer, and the helicity $\gamma$, where $\Phi(\varphi)=m \varphi+\gamma$. In what follows, we apply neutron scattering techniques which, in principle, allow the direct determination of any $\gamma$ value.

The bulk cubic chiral helimagnets with a B20 structure, such as $\mathrm{MnSi}$ and $\mathrm{FeGe}$, host whirlpool-like Bloch-type skyrmions described by $m=+1$ and $\gamma= \pm \pi / 2$ [4,11,12]. In these compounds the competition between a dominant ferromagnetic (FM) exchange and weaker DzyaloshinskiiMoriya interactions (DMIs) stabilizes a long-wavelength $(\sim 10-100 \mathrm{~nm})$ helical ground state in zero magnetic field $(B)$. The SkL phase exists under a finite $B$, where it competes with either a spin-flop conical phase or a field polarized state. Since

\footnotetext{
*jonathan.white@psi.ch

†bordacs.sandor@wigner.bme.hu
}

the SkL is stable typically only over the narrow temperature $(T)$ range $0.9 T_{C} \leqslant T \leqslant T_{C}$, where $T_{C}$ is the ordering $T$, thermal fluctuations are proposed to be crucial for stabilizing skyrmion phases [11-13].

Recently, a new bulk skyrmion phase was reported in the lacunar spinel $\mathrm{GaV}_{4} \mathrm{~S}_{8}$ (GVS) [14]. At high $T, \mathrm{GVS}$ has a rocksaltlike structure composed of alternating $\mathrm{GaS}_{4}$ and $\mathrm{V}_{4} \mathrm{~S}_{4}$ clusters. The highest-energy unpaired electron of the $\mathrm{V}_{4} \mathrm{~S}_{4}$ cluster occupies a triply degenerate molecular orbital which drives a cubic $(F \overline{4} 3 m)$ to rhombohedral $(R 3 m)$ Jahn-Teller transition at $T_{\mathrm{JT}}=42 \mathrm{~K}$ [15-19]. The loss of $\overline{4}$ symmetry below $T_{\mathrm{JT}}$ leads to the nucleation of four polar domains [15], each carrying an electric polarization along its rhombohedral axis, which lies parallel to one of the four cubic $\langle 111\rangle$ directions $[16,18,20]$. Below $T_{\mathrm{JT}}$, the Curie-Weiss $T$ indicates predominantly FM interactions between $S=1 / 2$ spins of $\mathrm{V}_{4} \mathrm{~S}_{4}$ clusters, and the system displays a strong easy-axis magnetic anisotropy [14,18]. In contrast to a uniform FM state, however, earlier small-angle neutron scattering (SANS) studies revealed that below $T_{C}=13 \mathrm{~K}$, long-wavelength spiral order develops with a periodicity of $17 \mathrm{~nm}$ at $T=11 \mathrm{~K}$ [14].

Due to the rhombohedral $C_{3 v}$ point symmetry of GVS at low $T$, the DMI pattern is expected to support a Néel-type SkL state with $m=+1$ and $\gamma=0$ or $\pi[11,14]$, instead of a Blochtype SkL. Correspondingly, the zero-field spiral state of GVS should be cycloidally and not helically modulated. However, microscopic experimental evidence for cycloidal order in GVS remains missing up to now. In addition, the skyrmion phase in GVS is reported to extend over a broad $T$ range down to $0.68 T_{C}$, while, surprisingly, the spiral order at zero field is stable only above $T_{S} \sim 5 \mathrm{~K}$, below which a FM state prevails. Hence, the key open questions concern the detailed nature of 

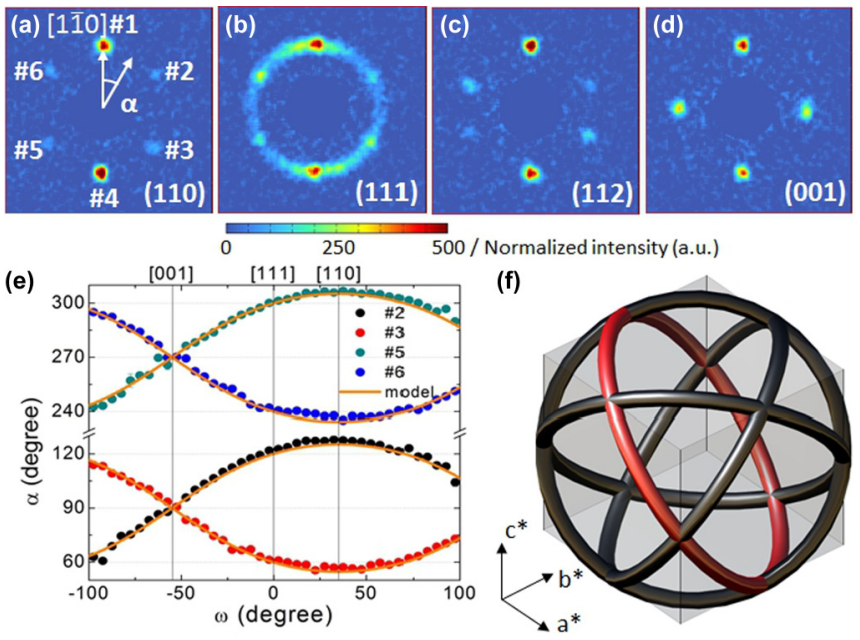

FIG. 1. Typical SANS data from GVS at $11 \mathrm{~K}$ and in $0 \mathrm{~T}$, and recorded in the (a) (110), (b) (111), (c) (112), and (d) (001) planes, respectively. (e) Angular position [ $\alpha$ as defined in (a)] of intensity spots $\# 2, \# 3$, \#5, and \#6 as a function of rocking angle $\omega$ around the vertical [1 $1 \overline{1} 0]$ axis. (f) Schematic image of the deduced intensity distribution in reciprocal space. An intensity ring confined to a $\{111\}$ plane appears in each rhombohedral domain, which describes well the positions of the spots (orange lines) in (e). The ring for the [111] domain is displayed in red only for clarity.

both the spiral order and phase stability in GVS, with this system attracting special interest as a hitherto unique bulk host of Néel-type skyrmions.

In this Rapid Communication we report comprehensive SANS studies that provide deeper insight into the magnetic order in GVS than reported earlier [14]. We show that the zerofield spiral state is indeed cycloidally modulated, underlining that in a finite field the Néel-type skyrmion state is formed. In striking contrast to the $B 20$ materials where a helical order forms the ground state as $T \rightarrow 0$, in GVS we observe the cycloidal order to collapse as the system becomes FM below $T_{S}=5 \mathrm{~K}$. We thus conclude GVS to display a delicate interplay between thermal fluctuations which stabilize all of the modulated states closer to $T_{C}$, and a strong easy-axis magnetic anisotropy that drives the formation of the FM state at low $T$.

Our SANS experiments were done using a $25 \mathrm{mg}$ single crystal of GVS, with the details of the crystal growth reported in Ref. [14]. Unpolarized SANS experiments were performed using the SANS-I instrument of the Paul Scherrer Institut and at D33 instrument of the Institut Laue-Langevin. Polarized SANS was also implemented at D33 as described in Ref. [21]. Full details of our experiment are given in the Supplemental Material [22].

First, unpolarized SANS was used to map the distribution of scattered intensity from the spiral phase of GVS at $T=$ $11 \mathrm{~K}$ and $B=0 \mathrm{~T}$. SANS data were collected over a broad range of angles as the crystal was rotated ("rocked") around its vertical [1 $1 \overline{1} 0]$ axis. Along with the two spots aligned with $[1 \overline{1} 0]$ and [ $\overline{1} 10]$ - which are on the rotation axis and so appear at all rocking angles-four other spots are also observed at all rocking angles with their relative angular separation changing smoothly [Figs. 1(a)-1(e)]. As shown in Figs. 1(b) and 1(e), in the (111) image plane the six spots form a regular hexagon, while upon rotating towards (001) the angle between spots \#2-\#5 and \#3-\#6 decreases and they eventually merge in the (001) plane to form a four-spot pattern [Fig. 1(d)].

Besides the six spots, a ring of intensity is also observed lying in the (111) and ( $\overline{11} 1)$ planes-see Fig. 1(b) for the (111) plane. The angular positions of the spot pairs \#2-\#5 and \#3-\#6 can also be ascribed to two additional intensity rings in the $(\overline{1} 1 \overline{1})$ and $(1 \overline{11})$ planes that appear as spots when intersecting the SANS detector. The rocking-angle dependence of the spot positions is consistently described by this model [see orange lines in Fig. 1(e)].

Importantly then, in GVS the magnetic intensity is not concentrated into Bragg spots, as suggested previously from SANS imaging done only close to certain planes [14]. Instead, our analysis of wide rocking-angle data [Fig. 1(e)] shows the intensity to form four homogeneous rings in reciprocal space, with all SANS images showing an intersection of the four rings with the detector [Figs. 1(a)-1(d)]. Each ring originates from one of the four polar domains that form below $T_{\mathrm{JT}}$, and has scattering vectors $\mathbf{q}$ confined to the plane normal to the associated rhombohedral axis, as shown in Fig. 1(f).

The assignment of intensity rings allows the selective analysis of the magnetic correlations within each of the four polar domains, even while the orientations of $\mathbf{q}$ are ill defined. This latter point indicates any anisotropy in the plane normal to the rhombohedral axis to be exceedingly weak. This partial order lies in stark contrast with B20 helimagnets, where the cubic anisotropy dictates rigid directions of magnetic propagation at all $T$ 's in zero field [12,23-25]. The order seen in GVS is more reminiscent of liquid crystals, but instead of fluctuations of molecular orientations in real space, here the orientational disorder is reflected by a broad $q$ distribution.

To study the helicity state of the spiral order in detail, polarized SANS experiments were done at $T=11 \mathrm{~K}$. A small, $B=10 \mathrm{mT}$ magnetic guide field was applied along [111] to maintain a longitudinal neutron spin polarization axis parallel to the beam, and which leaves intact the cycloidal phase in all polar domains [14]. The relevant terms contributing to the scattered intensities in the non-spin-flip (NSF) and spin-flip (SF) processes are [26]

$$
\begin{aligned}
& I_{\mathrm{NSF}}^{ \pm \pm} \propto\left|N(\mathbf{q}) \pm m_{z}^{\perp}(\mathbf{q})\right|^{2}, \\
& I_{\mathrm{SF}}^{ \pm \mp} \propto\left|m_{x}^{\perp}(\mathbf{q}) \pm i m_{y}^{\perp}(\mathbf{q})\right|^{2},
\end{aligned}
$$

where $m_{x}^{\perp}(\mathbf{q}), m_{y}^{\perp}(\mathbf{q})$, and $m_{z}^{\perp}(\mathbf{q})$ are the $x, y$, and $z$ components of the Fourier transform of the local magnetization normal to $\mathbf{q}$, and $N(\mathbf{q})$ is the Fourier transform of the nuclear scattering-length density. Here, we define the Cartesian $z$ axis as parallel to the neutron spin polarization axis $\left(\mathbf{P}_{z}\right)$. According to these equations, the NSF intensity is due to both nuclear scattering, and magnetic scattering from spin components that are simultaneously $\| z$ and normal to $\mathbf{q}$. In contrast, the SF intensity can be assigned exclusively to magnetic scattering from spin components normal to both $z$ and $\mathbf{q}$.

Figures 2(a) and 2(b) respectively show the NSF and SF scattering patterns observed in the (111) plane. The magnetic scattering ring from the [111] structural domain (with rhombohedral axis $\| z$ for $T<T_{\mathrm{JT}}$ ), contributes exclusively to NSF scattering, while the scattering rings from the other three polar 


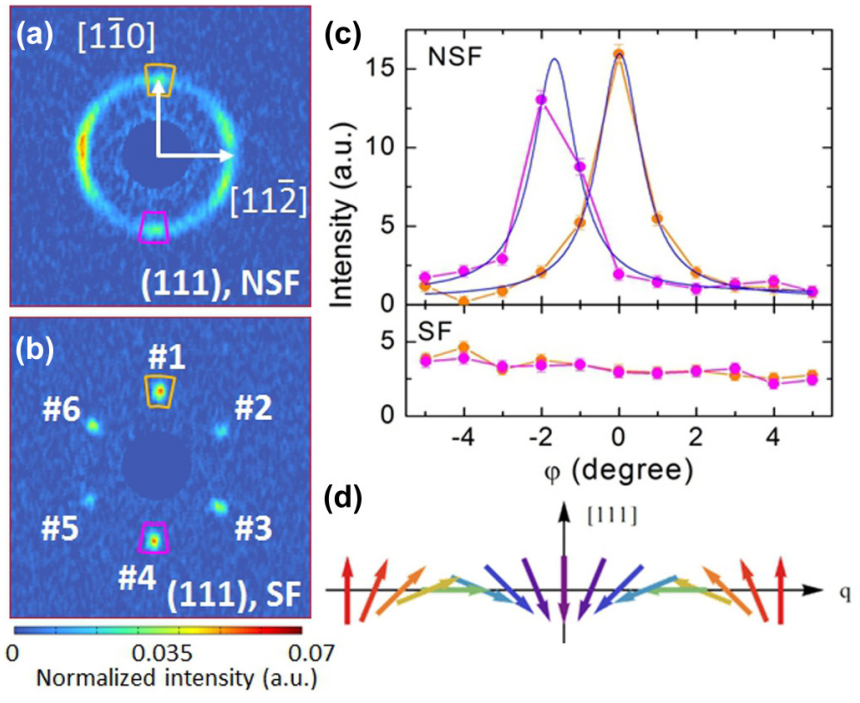

FIG. 2. (a) Non-spin-flip (NSF) and (b) spin-flip (SF) SANS scattering patterns measured on the (111) plane at $11 \mathrm{~K}$ in $10 \mathrm{mT}$. (c) Rocking curves for the orange and magenta boxes shown in (a) and (b) for a sample rotation around the horizontal, [11 2 ] axis. (d) Schematic of a regular cycloidal magnetic structure with spins rotating in a plane including $\mathbf{q}$.

domains contribute to both NSF and SF scattering. The appearance of the scattering ring from the [111] structural domain only in the NSF channel confirms its origin as due to spin components $\| z$, and so the rhombohedral axis. The absence of SF scattering from this structural domain rules out further spin components normal to both $\mathbf{q}$ and $z$, which would be finite for helical order. Instead, the data are consistent with scattering from a cycloid magnetic order, where moments rotate within a plane defined by $\mathbf{q}$ and the rhombohedral axis [Fig. 2(d)].

Figure 2(c) shows polarized SANS rocking curves for

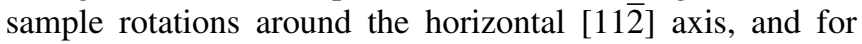
intensity regions defined by the orange and magenta boxes in Figs. 2(a) and 2(b). These data show clearly that the complete ring of scattering in the (111) plane is solely responsible for the peak in the NSF intensity. From Lorentzian peak fits, the correlation length along the rhombohedral axis is $\xi_{\perp}=2 /(\mathrm{FWHM} \cdot|q|) \sim 170 \mathrm{~nm}$, where FWHM is the full width at half maximum of the peak [27]. The value of $\xi_{\perp}$ is comparable with that reported for high-purity $\mathrm{MnSi}$ samples [12], indicating well-developed magnetic order along the rhombohedral axis.

From the other three polar domains with rhombohedral axes spanning $71^{\circ}$ with $\mathbf{P}_{z}$, the rocking curves trace the intensity rings out of the detector plane, and so the magnetic scattering is always finite, and almost rocking-angle independent. In the NSF rocking curve, it forms a baseline underneath the peak, while in the SF rocking curve, only the baseline is observed. The relative ratio of the baseline NSF:SF scattering is estimated to be $\sim 0.17$, which is in relatively good agreement with the ratio of 1:8 expected in accord with a regular cycloid model [Fig. 2(d)]. The cycloidal nature of the spin correlations is further supported by spin-polarized experiments with $\mathbf{P}_{z} \|$ [110] (see Supplemental Material [22]). Since the cycloidal order emerges as a consequence of the pattern of the DMI

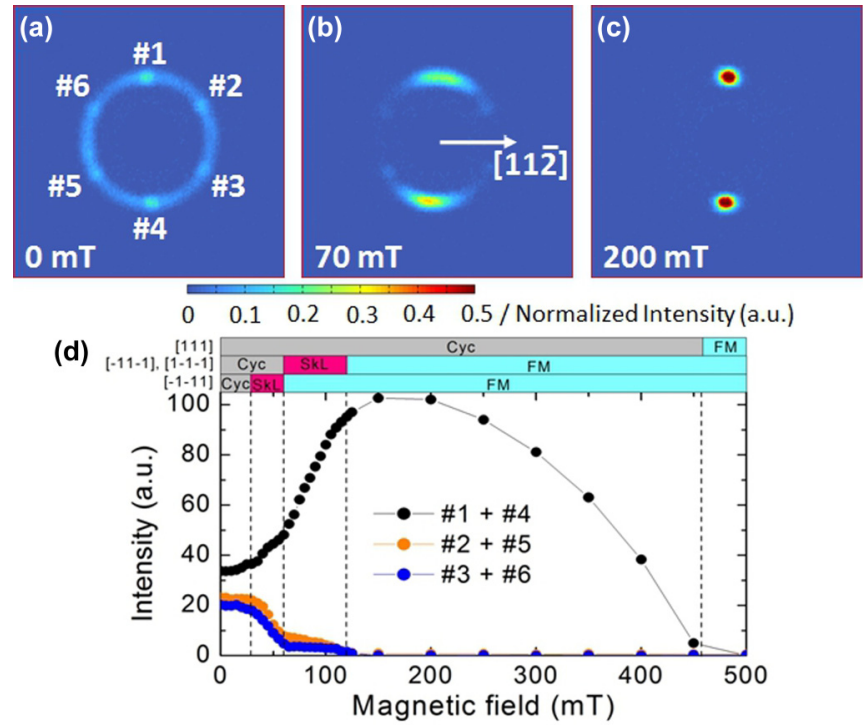

FIG. 3. SANS patterns measured at $11 \mathrm{~K}$ in the (111) plane at (a) 0, (b) 70, and (c) $200 \mathrm{mT}$, when $\mathbf{B} \|$ [11 $\overline{2}]$. (d) $B$ dependence of the SANS intensity for the positions defined in (a). Phase diagrams for each polar domain are shown and labeled according to the orientation of the rhombohedral axis, with the dashed lines denoting phase boundaries discussed in Ref. [14].

couplings specific for the low $T C_{3 v}$ polar crystal symmetry of GVS [14], the result strongly suggests that the skyrmion state in finite field can only be of the Néel type and not the Bloch type. Indeed, the same distinction was found between the SF and NSF channel in the SkL phase of GVS (see Supplemental Material [22]), which confirms the skyrmion state to be formed by cycloids in GVS.

As further evidence in support of the cycloid model, in Figs. 3(a)-3(c) we show unpolarized SANS data obtained from the (111) plane when a transverse $B \|[11 \overline{2}]$ was applied normal to the rhombohedral axis arising from the [111] domain. After zero-field cooling [Fig. 3(a)], a complete scattering ring is observed from the [111] domain, along with spots at locations $\# 1-\# 4$, \#2-\#5, and \#3-\#6 due to scattering rings from the three remaining polar domains with rhombohedral axes along

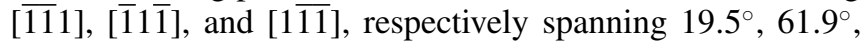
and $61.9^{\circ}$ with $B$. In these three domains, the component of $B$ along the respective rhombohedral axis drives the cycloidalskyrmion and skyrmion-FM phase transitions, as indicated by the magnetic phase diagrams shown in Fig. 3(d) [14].

In contrast, for the [111] domain, increasing $B$ causes the intensity distributed around the ring to gradually collect around the positions \#1-\#4 and form a spot pair with $\mathbf{q}$ normal to $B$ [Figs. 3(b) and 3(c)] that survives up to high field [Fig. 3(d)]. This provides further evidence that cycloidal order is realized in GVS, since the susceptibility of a cycloid is generally enhanced normal to the cycloid plane containing q. Under an in-plane $B$, the cycloid order transforms smoothly into a transverse conical order with $\mathbf{q}$ normal to $B$. Here, the cone axis is parallel to $B$, and the cone angle closes at $\sim 0.45 \mathrm{~T}$ [Fig. 3(d)]. Combining these data with previous experimental [14] and theoretical [28] results shows that in GVS the transverse conical-type state exists only when $B$ lies close $\left(\lesssim 19^{\circ}\right)$ to the plane within which 

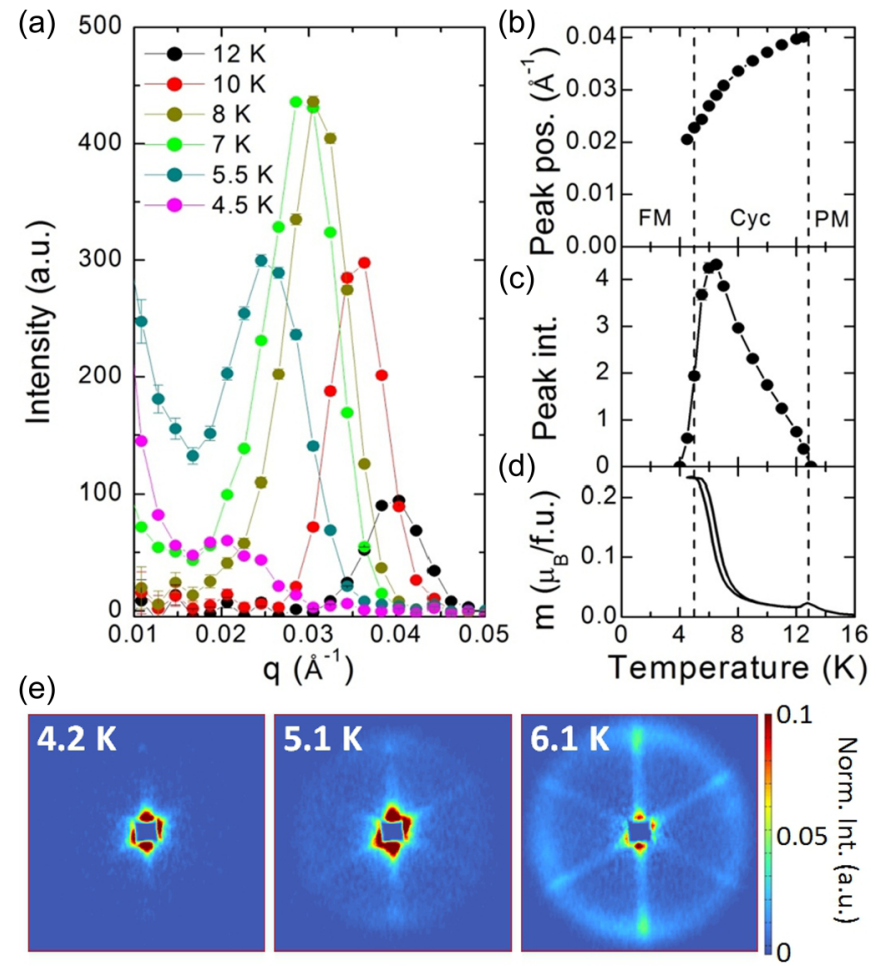

FIG. 4. (a) $T$ dependence of the $|q|$-dependent, azimuthally averaged SANS intensity on the detector plane. (b) $T$ dependence of the cycloid scattering vector and (c) its intensity. (d) $T$ dependence of the magnetization measured in $10 \mathrm{mT}$. (e) Typical unpolarized SANS patterns measured in the vicinity of the ferromagnetic-cycloidal transition.

$\mathbf{q}$ is confined. This lies in contrast to cubic helimagnets where $B, \mathbf{q}$, and the cone axis are all parallel with each other, and this longitudinal conical phase covers the major part of the phase diagram for any $B$ direction [12,24,25].

Figure 4 shows unpolarized SANS data from GVS summarizing the $T$ evolution of the cycloidal order at $B=0 \mathrm{~T}$. The smooth increase of SANS intensity at finite $|q|$ upon cooling below $T_{C}=13 \mathrm{~K}$ [Fig. 4(c)] implies a continuous transition between the paramagnetic and cycloidal states. Figures 4(a) and 4(b) show the intensity to peak initially at $|q|=0.04 \AA^{-1}$, and $|q|$ to fall by $50 \%$ on cooling. In the low- $T$ range where $|q|$ is still finite, the intensity drops sharply to zero [Fig. 4(c)] and simultaneously a tail of low- $q$ scattering develops, indicating growing FM correlations [Fig. 4(a)]. This phase coexistence, sharp change in SANS intensity, and hysteresis in the $T$ dependence of the magnetization [Fig. 4(d)] indicate the cycloidal-FM transition to be first order, consistent with recent specific heat data [18]. In contrast, however, Fig. 4(a) shows that close to $T_{S}$ the radial intensity distribution has significantly broadened in $|q|$, and the intensity ring has transformed into a disklike profile in the (111) plane with a sharp cut off at the high- $q$ end [Fig. 4(e)]. In the other three polar domains, the intensity disks appear as a six-spoked asterisk when imaged by SANS along the [111] axis. These data show the length of $|q|$ to vary strongly, or even fluctuate, on the approach to $T_{S}$. This could indicate the transition at $T_{S}$ to be continuous in theory, but hysteresis effects emerge due to slow dynamics when the rearrangements of large structures are involved [29], as also found recently in MnSi [25].

Nevertheless, in comparison with the general phase diagram of the $B 20$ helimagnets, the results reported here for GVS stand out as rather unusual. At such low $T$ s in GVS, it is unlikely that the microscopic couplings such as the symmetric exchange or DMIs vary sufficiently to drive the cycloidal-FM transition at $T_{S}$. A more plausible scenario is that thermal fluctuations are crucial for stabilizing the long-wavelength modulated states close to $T_{C}$, and when they are suppressed at low $T$, the system transforms to a FM state, expected in accord with the strong easy-axis magnetic anisotropy [14].

Similar behavior has been discussed in the context of the spiral-FM transition in the elemental rare-earth metals such as $\mathrm{Dy}$ and $\mathrm{Tb}$, wherein the effective anisotropy becomes more influential as fluctuations are suppressed at low $T$ [30]. In those systems the strong axial anisotropy leads to a larger periodicity in real space (shorter $|q|$ ) with increased anharmonicity, and close to the transition the spiral transforms into a soliton lattice with periodically arranged FM domain walls. Such a state in GVS may explain the unusual $T$-dependent SANS data shown in Fig. 4(e). Indeed, the reduction of $|q|$ by $50 \%$ on cooling from $T_{C}$ to $T_{S}$ [Fig. 4(b)] supports this picture, although close to $T_{S}$ the signal expected at higher-order $q$ due to soliton lattice formation lies below the detectable limit. Nevertheless, the proposed $T$-dependent interplay between the easy-axis anisotropy and thermal fluctuations likely governs the fine structure of the phase diagram in GVS, again in contrast with the $B 20$ s which have a comparatively weaker anisotropy, and in which $|q|$ changes at most $10 \%$ upon cooling towards a low- $T$ ground state that always remains helically modulated [24].

To summarize, from SANS studies of $\mathrm{GaV}_{4} \mathrm{~S}_{8}$ (GVS) in zero magnetic field $(B)$ we observe the cycloidal order close to $T_{C}$ to give way to a FM ground state as $T \rightarrow 0$. This suggests thermal fluctuations to be crucial for stabilizing the modulated states in GVS, while ferromagnetism emerges at low $T$ due to the strong easy-axis magnetic anisotropy. Using polarized SANS, we showed that the skyrmion helicity in GVS is consistent only with the Néel type. The technique can be readily applied for characterizing skyrmionic spin textures in noncentrosymmetric magnets of other point symmetries, such as the recently proposed antiskyrmion state in a $D_{2 d}$ magnet composed of coexisting helical and cycloidal modulations [31].

We wish to thank N. Reynolds, L. Rózsa, U. K. Rössler, M. Markó, and, in particular, I. Kézsmárki, A. Loidl, and H. M. Rønnow for fruitful discussions and support. This work was supported by Hungarian Research Funds No. OTKA K 108918, No. OTKA PD 111756, No. TKA-DAAD 152294, and No. Bolyai 00565/14/11, by the Lendület Program of the Hungarian Academy of Sciences, the Swiss National Science Foundation Sinergia network NanoSkyrmionics, and the Swiss National Science Foundation (SNF) Project Grant No. 153451. A.B. was supported by the ÚNKP-17-3-III New National Excellence Program of the Ministry of Human Capacities. This work is based on neutron experiments performed at the Swiss Spallation Neutron Source SINQ, Paul Scherrer Institute (PSI), Villigen, Switzerland, and the Institut Laue-Langevin (ILL), Grenoble, France. 
[1] P. Milde, D. Köhler, J. Seidel, L. M. Eng, A. Bauer, A. Chacon, J. Kindervater, S. Mühlbauer, C. Pfleiderer, S. Buhrandt, C. Schütte, and A. Rosch, Science 340, 1076 (2013).

[2] N. Romming, C. Hanneken, M. Menzel, J. E. Bickel, B. Wolter, K. von Bergmann, A. Kubetzka, and R. Wiesendanger, Science 341, 636 (2013).

[3] W. Koshibae and N. Nagaosa, Nat. Commun. 5, 5148 (2014).

[4] X. Z. Yu, Y. Onose, N. Kanazawa, J. H. Park, J. H. Han, Y. Matsui, N. Nagaosa, and Y. Tokura, Nature (London) 465, 901 (2010).

[5] K. Karube, J. S. White, N. Reynolds, J. L. Gavilano, H. Oike, A. Kikkawa, F. Kagawa, Y. Tokunaga, H. M. Rønnow, Y. Tokura, and Y. Taguchi, Nat. Mater. 15, 1237 (2016).

[6] F. Jonietz, S. Mühlbauer, C. Pfleiderer, A. Neubauer, W. Münzer, A. Bauer, T. Adams, R. Georgii, P. Böni, R. A. Duine, K. Everschor, M. Garst, and A. Rosch, Science 330, 1648 (2010).

[7] X. Z. Yu, N. Kanazawa, W. Z. Zhang, T. Nagai, T. Hara, K. Kimoto, Y. Matsui, Y. Onose, and Y. Tokura, Nat. Commun. 3, 988 (2012)

[8] N. Nagaosa and Y. Tokura, Nat. Nanotechnol. 8, 899 (2013).

[9] J. Sampaio, V. Cros, S. Rohart, A. Thiaville, and A. Fert, Nat. Nanotechnol. 8, 839 (2013).

[10] X. Zhang, M. Ezawa, and Y. Zhou, Sci. Rep. 5, 9400 (2015).

[11] A. N. Bogdanov and D. A. Yablonskii, Sov. Phys. JETP 68, 101 (1989); U. K. Rössler, A. N. Bogdanov, and C. Pfleiderer, Nature (London) 442, 797 (2006).

[12] S. Mühlbauer, B. Binz, F. Jonietz, C. Pfleiderer, A. Rosch, A. Neubauer, R. Georgii, and P. Böni, Science 323, 915 (2009).

[13] M. Janoschek, M. Garst, A. Bauer, P. Krautscheid, R. Georgii, P. Böni, and C. Pfleiderer, Phys. Rev. B 87, 134407 (2013).

[14] I. Kézsmárki, S. Bordács, P. Milde, E. Neuber, L. M. Eng, J. S. White, H. M. Rønnow, C. D. Dewhurst, M. Mochizuki, K. Yanai, H. Nakamura, D. Ehlers, V. Tsurkan, and A. Loidl, Nat. Mater. 14, 1116 (2015).

[15] R. Pocha, D. Johrendt, and R. Pöttgen, Chem. Mater. 12, 2882 (2000).

[16] E. Ruff, S. Widmann, P. Lunkenheimer, V. Tsurkan, S. Bordács, I. Kézsmárki, and A. Loidl, Sci. Adv. 1, e1500916 (2015).

[17] Z. Wang, E. Ruff, M. Schmidt, V. Tsurkan, I. Kézsmárki, P. Lunkenheimer, and A. Loidl, Phys. Rev. Lett. 115, 207601 (2015).
[18] S. Widmann, E. Ruff, A. Günther, H.-A. K. von Nidda, P. Lunkenheimer, V. Tsurkan, S. Bordács, I. Kézsmárki, and A. Loidl, Philos. Mag. 97, 3428 (2016).

[19] J. Hlinka, F. Borodavka, I. Rafalovskyi, Z. Docekalova, J. Pokorny, I. Gregora, V. Tsurkan, H. Nakamura, F. Mayr, C. A. Kuntscher, A. Loidl, S. Bordács, D. Szaller, H.-J. Lee, J. H. Lee, and I. Kézsmárki, Phys. Rev. B 94, 060104(R) (2016).

[20] A. Butykai, S. Bordács, I. Kézsmárki, V. Tsurkan, A. Loidl, J. Döring, E. Neuber, P. Milde, S. C. Kehr, and L. M. Eng, Sci. Rep. 7, 44663 (2017).

[21] C. D. Dewhurst, I. Grillo, D. Honecker, M. Bonnaud, M. Jacques, C. Amrouni, A. Perillo-Marcone, G. Manzin, and R. Cubitt, J. Appl. Cryst. 49, 1 (2016).

[22] See Supplemental Material at http://link.aps.org/supplemental/ 10.1103/PhysRevB.97.020401 for full details of the polarized and unpolarised small-angle neutron scattering (SANS) experiments, and further supporting polarized SANS data.

[23] S. V. Grigoriev, S. V. Maleyev, A. I. Okorokov, Y. O. Chetverikov, R. Georgii, P. Böni, D. Lamago, H. Eckerlebe, and K. Pranzas, Phys. Rev. B 72, 134420 (2005).

[24] S. V. Grigoriev, S. V. Maleyev, A. I. Okorokov, Y. O. Chetverikov, P. Böni, R. Georgii, D. Lamago, H. Eckerlebe, and K. Pranzas, Phys. Rev. B 74, 214414 (2006).

[25] A. Bauer, A. Chacon, M. Wagner, M. Halder, R. Georgii, A. Rosch, C. Pfleiderer, and M. Garst, Phys. Rev. B 95, 024429 (2017).

[26] R. M. Moon, T. Riste, and W. C. Koehler, Phys. Rev. 181, 920 (1969).

[27] The in-plane radial correlation length of the ring appearing in the (111) plane is estimated to be $\xi_{q} \sim 40 \mathrm{~nm}$ as determined from the $I$ vs $|q|$ plot, and after deconvoluting the instrumental broadening.

[28] A. O. Leonov and I. Kézsmárki, Phys. Rev. B 96, 214413 (2017).

[29] Á. Butykai, S. Bordács, L. F. Kiss, B. Gy. Szigeti, V. Tsurkan, A. Loidl, and I. Kézsmárki, Phys. Rev. B 96, 104430 (2017).

[30] Y. A. Izyumov, Sov. Phys. Usp. 27, 845 (1984).

[31] A. K. Nayak, V. Kumar, T.-P. Ma, P. Werner, E. Pippel, R. Sahoo, F. Damay, U. K. Rößler, C. Felser, and S. S. P. Parkin, Nature (London) 548, 561 (2017). 